ESAIM: PROCEEDINGS AND SURVEYS, September 2014, Vol. 45, p. 32-47

J.-S. Dhersin, Editor

\title{
MARTINGALE INEQUALITIES, OPTIMAL MARTINGALE TRANSPORT, AND ROBUST SUPERHEDGING*
}

\author{
NizAR TOUZI ${ }^{1}$
}

\begin{abstract}
In the recent literature, martingale inequalities have been emphasized to be induced by pathwise inequalities independently of any reference probability measure on the paths space. This feature is closely related to the problem of robust hedging in financial mathematics, which was originally addressed in some specific cases by means of the Skorohod embedding problem. The martingale optimal transport problem provides a systematic framework for the robust hedging problem and, therefore, allows to derive sharp martingale inequalities. We illustrate this methodology by deriving the sharpest possible control of the running maximum of a martingale by means of a finite number of marginals.
\end{abstract}

\section{INTRODUCTION}

This paper summarizes my plenary talk at the SMAI Congress, Seignosse 2013. The objective is to show recent connections between martingale inequalities and the problem of robust hedging in financial mathematics.

Following the seminal paper by Hobson [18], the problem of robust hedging was addressed by exploiting the connection with the so-called Skorohod embedding problem, see Hobson [20] and Obloj [26] for an overview. In the context of some specific problems of hedging, Hobson [18] used the Azéma-Yor [2,3] solution of the Skorohod embedding problem to derive the optimal bounds on the no-arbitrage valuation of such contracts, and showed that they correspond to model-free hedging strategies which are exhibited explicitly. As a by-product of the last results, [18] obtains a family of sharp martingale inequalities controlling the running maximum of a martingale by the marginal distribution of its final value. Brown, Hobson and Rogers [7] extend these results to the two-marginals case, building on a remarkable pathwise inequality which turns out to provide sharp martingale inequalities. We also mention the papers $[1,8,10-14,21-23]$ which follow the same line of thinking.

The previous literature raises two important questions. First, how specific is the finding by Hobson that Doob's type martingale inequalities is induced by a pathwise inequality? Second, how to guess the pathwise inequalities so that the induced martingale inequalities are sharp, i.e. hold with equality for some martingale?

The first question was answered recently by Bouchard and Nutz [9] who proved that any martingale inequality is induced by a pathwise inequality.

The second question was also answered recently by the newly introduced problem of martingale optimal transport. A general formulation of the problem of robust hedging was recently introduced by Beiglböck, HenryLabordère, and Penkner [4] in discrete-time, and Galichon Henry-Labordère and Touzi [16] in continuous-time. This formulation opens a new problem of optimal transport. See Tan and Touzi [29], Dolinsky and Soner [15], and the numerical approximation by Bonnans and Tan [5]. Finally, Galichon, Henry-Labordère and Touzi [16], and Henry-Labordère, Obloj, Spoida, and Touzi [17] illustrate how the martingale optimal transport approach

\footnotetext{
* The work of the author is supported by the ERC grant RoFiRm 321111.

${ }^{1}$ Ecole Polytechnique Paris, Centre de Mathématiques Appliquées, nizar.touzi@polytechnique.edu.
}

(C) EDP Sciences, SMAI 2014 
allows to derive the sharp pathwise inequalities. The latter problem also opened the door for a multimarginals extension, by Obloj and Spoida [27], of the Azéma-Yor solution of the Skorohod embedding problem.

The paper is organized as follows.Section 2 recalls briefly the Skorohod embedding problem together with the corresponding Azéma-Yor solution, and the connection to the problem of model-free hedging. Sections 3 and 4 review the known pathwise inequalities which induce a Doob's type martingale inequality. Section 5 introduces the martingale optimal transport as a general formulation of the problem of robust hedging. Finally, Section 6 report some applications which illustrate the effectiveness of the martingale transport approach to derive the sharp pathwise inequalities inducing sharp martingale inequalities.

\section{Skorohod Embedding and Robust Superhedging}

\subsection{The Skorohod embedding problem}

Let $W$ be a Brownian motion, and $\mu$ a centered probability measure on $\mathbb{R}$. The Skorohod embedding problem is:

$\operatorname{SEP}(\mu)$ Find a stopping time $\tau$ such that $W_{\tau} \sim \mu$ and $\left\{W_{t \wedge \tau}\right\}_{t \geq 0}$ UI martingale,

where UI is an abbreviation of "niformly integrable".

Doob's solution To start with, let us forget about the uniform integrability requirement, and provide an easy example of a stopping time $\tau$ such that $W_{\tau}$ has distribution $\mu$. As usual, we denote $F_{\mu}(x):=\mu((-\infty, x])$ and $F_{\mathcal{N}}:=F_{\mathcal{N}(0,1)}$. Define

$$
\tau:=\inf \left\{t \geq 1: W_{t}=F_{\mu}^{-1} \circ F_{\mathcal{N}}\left(W_{1}\right)\right\}
$$

where $F_{\mu}^{-1}$ is the right-continuous inverse function of $F_{\mu}$. Then $W_{\tau}=F_{\mu}^{-1} \circ F_{\mathcal{N}}\left(W_{1}\right) \sim \mu$.

However, we observe that $\left\{W_{t \wedge \tau}\right\}_{t \geq 0}$ is not uniformly integrable. Indeed, assuming to the contrary that it is UI, we would obtain by the martingale property that $F_{\mu}^{-1} \circ F_{\mathcal{N}}\left(W_{1}\right)=W_{\tau}=\mathbb{E}\left[W_{\tau} \mid W_{1}\right]=W_{1}$, a.s., which can not happen except in the trivial case $\mu=\mathcal{N}(0,1)$.

\subsection{The Azema-Yor solution of the Skorohod embedding problem}

For our future developments, we recall a particular solution of the Skorohod embedding problem, which will be the driving example for the connection with the martingale optimal transport. We therefore focus on the detailed presentation of this particular solution. The crucial ingredient for the Azéma-Yor construction is the barycenter function of a probability measure $\mu \in \mathcal{P}_{\mathbb{R}}$ defined by

$$
b_{\mu}(x):=\mathbf{1}_{\{\mu([x, \infty)>0\}} \frac{\int_{[x, \infty)} y \mu(d y)}{\int_{[x, \infty)} \mu(d y)}+x \mathbf{1}_{\{\mu([x, \infty)=0\}}, \quad \text { for all } \quad x \in \mathbb{R} .
$$

Theorem 2.1. Let $B$ be a Brownian motion on $(\Omega, \mathcal{F}, \mathbb{P})$, denote by $B^{*}$ the corresponding running maximum process, and let $\mu \in \mathcal{P}_{\mathbb{R}}$ be a probability measure on $\mathbb{R}$. Consider the stopping time $\theta_{\mu}:=\inf \left\{t \geq 0: B_{t}^{*} \geq\right.$ $\left.b_{\mu}\left(B_{t}\right)\right\}$.

Then $B_{\theta_{\mu}} \sim \mu$, and $B_{. \wedge \theta_{\mu}}$ is a uniformly integrable martingale.

\subsection{Connection with robust hedging}

We now show that there is a one-to-one correspondence between the Skorohod embedding problem and the choice of an arbitrage-free model with given marginal constraint. 
Financial market Consider a financial market consisting of a non risky asset with price process normalized to unity, and a risky asset with price process $X=\left\{X_{t}, t \geq 0\right\}$.

Let $T>0$ be some given maturity. We also assume that the financial market allows for the trading $T$-maturity European call and put options, with all possible strikes, defined respectively by the payoffs at maturity $T$ :

$$
\left(X_{T}-K\right)^{+} \text {and }\left(K-X_{T}\right)^{+}, \quad K \in \mathbb{R}
$$

The time zero price of the $(T, K)$ call and put options are known and given respectively by $c_{X}(K)$ and $p_{X}(K)$, which are assumed to satisfy the so-called call-put parity:

$$
c_{X}(K)-p_{X}(K)=c_{Y}(K)-p_{Y}(K)=X_{0}-K, \quad \text { for all } \quad K \in \mathbb{R} .
$$

Moreover, by no arbitrage considerations, it follows that these prices are convex and can be written in terms of a probability measure $\mu$ with mean $X_{0}$ :

$$
c_{X}(K)=\int(x-K)^{+} \mu(d x) \text { and } p_{X}(K)=\int(K-x)^{+} \mu(d x) \quad \text { where } \quad \mu:=c_{X}^{\prime \prime}=p_{X}^{\prime \prime} .
$$

In other words, as noticed by Breeden and Litzenberger [6], the observation of $T$-maturity call and put prices, for all strikes, determines the marginal distribution $X_{T} \sim \mu$.

Notice that any smooth function $g$ can be decomposed in terms of calls and puts payoffs

$$
g\left(X_{T}\right)=g\left(x^{*}\right)+\left(X_{T}-x^{*}\right) g^{\prime}\left(x^{*}\right)+\int_{-\infty}^{x^{*}}\left(K-X_{T}\right)^{+} g^{\prime \prime}(K) d K+\int_{x^{*}}^{\infty}\left(X_{T}-K\right)^{+} g^{\prime \prime}(K) d K
$$

Then, assuming that market prices are linear and continuous, we deduce that the price of the payoffs $g\left(X_{T}\right)$ is:

$$
g\left(x^{*}\right)+\left(X_{0}-x^{*}\right) g^{\prime}\left(x^{*}\right)+\int_{-\infty}^{x^{*}} p_{X}(K) g^{\prime \prime}(K) d K+\int_{x^{*}}^{\infty} c_{X}(K) g^{\prime \prime}(K) d K=\int g(K) \mu(d K)
$$

where the last equality follows from two integrations by parts. Consequently by the assumed continuity of prices, we see that the previous pricing formula holds for any $\mu$-integrable functions $g$.

Martingale with given marginal The above financial market allows for the dynamic continuous-time trading of the non risky asset and the risky one, and for the static trading of european calls and puts with maturity $T$. Then, the no-arbitrage condition is essentially equivalent to the existence of a martingale measure with given $T$-marginal $\mu$ :

$$
X \quad \text { is a martingale with } \quad X_{T} \sim \mu \text {. }
$$

Finally, throughout this note, we assume that the process $X$ has continuous sample paths.

Connection with the Skorohod embedding problem By the Dubins-Schwartz time change theorem, it follows that $X_{t}-X_{0}=W_{\langle X\rangle_{t}}, t \leq T$, for some Brownian motion $W$, and $\tau:=\langle X\rangle_{T}$ is a stopping time with respect to the time changed filtration. Consequently $\tau$ is a solution of the $\operatorname{SEP}(\mu)$.

Conversely, let $W$ be a Brownian motion, and let $\tau$ be a solution of $\operatorname{SEP}(\mu)$. Define

$$
X_{t}:=X_{0}+B_{\frac{t}{T-t} \wedge \tau}, \quad t \leq T
$$

Then, $X$ is a continuous martingale, starting from $X_{0}$, with $X_{T} \sim \mu$. 
References The connection between the Skorohod embedding problem and the model-free hedging in finance was introduced by Hobson [18] in the context of Lookback options, i.e. derivative contract with payoff defined as a function of the running maximum of the underlying asset. We refer to Obloj [26] for a survey on the Skorohod embedding problem collecting more than twenty known solutions. We also refer to the lecture notes by Hobson [20] for an overview of the subject with link to the model-free hedging problem and applications to various examples of path-dependent derivatives.

\section{Pathwise martingale inequalities and Robust hedging of Lookback options}

In this section, we focus on the problem of model-free superhedging of Lookback options. A Lookback option is a derivative contract with payoff at maturity $T$ :

$$
\xi=\phi\left(X_{T}^{*}\right), \quad X_{T}^{*}:=\max _{t \in[0, T]} X_{t},
$$

where $\phi: \mathbb{R} \longrightarrow \mathbb{R}$ is a measurable payoff function. Our main result applies for non-decreasing functions $\phi$. The current approach was initiated by Hobson [18], and is based on the following pathwise inequality with elementary proof.

Lemma 3.1. Let $\omega: \mathbb{R}_{+} \longrightarrow \mathbb{R}$ be an arbitrary càdlàg function, and denote $\omega_{t}^{*}:=\sup _{s \leq t} \omega_{s}$. Then:

$$
\mathbf{1}_{\left\{\omega_{T}^{*} \geq m\right\}} \leq \frac{\left(\omega_{T}-\zeta\right)^{+}}{m-\zeta}+\frac{m-\omega_{T}}{m-\zeta} \mathbf{1}_{\left\{\omega_{T}^{*} \geq m\right\}} \quad \text { for all } \quad \zeta<m,
$$

and for all nondecreasing function $\phi$ :

$$
\phi\left(\omega_{T}^{*}\right) \leq \phi\left(\omega_{0}\right)+\int_{\omega_{0}}^{\infty}\left(\frac{\left(\omega_{T}-\zeta_{m}\right)^{+}}{m-\zeta_{m}}+\frac{m-\omega_{T}}{m-\zeta_{m}} \mathbf{1}_{\left\{\omega_{T}^{*} \geq m\right\}}\right) d \phi(m)
$$

for all $m \in \mathbb{R}$ and $\zeta_{m}<m$.

Proof. To verify the first inequality, notice that

$$
\begin{aligned}
\mathbf{1}_{\left\{\omega_{T}^{*} \geq m\right\}}-\frac{m-\omega_{T}}{m-\zeta} \mathbf{1}_{\left\{\omega_{T}^{*} \geq m\right\}} & =\frac{\omega_{T}-\zeta}{m-\zeta} \mathbf{1}_{\left\{\omega_{T}^{*} \geq m\right\}} \\
& \leq \frac{\left(\omega_{T}-\zeta\right)^{+}}{m-\zeta} \mathbf{1}_{\left\{\omega_{T}^{*} \geq m\right\}} \leq \frac{\left(\omega_{T}-\zeta\right)^{+}}{m-\zeta} .
\end{aligned}
$$

The second (more general) inequality follows from the observation that:

$$
\phi\left(\omega_{T}^{*}\right)=\phi\left(\omega_{0}\right)+\int_{\omega_{0}}^{\infty} \mathbf{1}_{\left\{\omega_{T}^{*} \geq m\right\}} d \phi(m)
$$

so that, given the non-decrease of $\phi$, the required inequality follows from the first one.

As an immediate consequence of the last pathwise inequality, we obtain the following upper bound for the no-arbitrage prices of Lookback options $\phi\left(X_{T}^{*}\right)$ with nondecreasing payoff function $g$.

Corollary 3.2. Let $X$ be a càdlàg submartingale. Then, for all $\zeta<m$ :

$$
\mathbb{E}\left[\mathbf{1}_{\left\{X_{T}^{*} \geq m\right\}}\right] \leq \frac{\mathbb{E}\left[\left(X_{T}-\zeta\right)^{+}\right]}{m-\zeta} \text { for all } \quad \zeta<m
$$


and for all nondecreasing $\phi: \mathbb{R} \longrightarrow \mathbb{R}$, we have

$$
\mathbb{E}\left[\phi\left(X_{T}^{*}\right)\right] \leq \phi\left(X_{0}\right)+\int_{X_{0}}^{\infty} \frac{\mathbb{E}\left[\left(X_{T}-\zeta_{m}\right)^{+}\right]}{m-\zeta_{m}} d \phi(m) \quad \text { for all } \quad \zeta<m
$$

Proof. As in the previous proof, given the non-decrease of $\phi$, the second inequality is immediately obtained from the first one. To prove the first inequality, we start from the pathwise inequality of Lemma 3.1, and we take expected values on both sides. This provides for all $\zeta<m$ :

$$
\mathbb{E}\left[\mathbf{1}_{\left\{X_{T}^{*} \geq m\right\}}\right] \leq \frac{\mathbb{E}\left[\left(X_{T}-\zeta\right)^{+}\right]}{m-\zeta}+\frac{\mathbb{E}\left[\left(m-X_{T}\right) \mathbf{1}_{\left\{X_{T}^{*} \geq m\right\}}\right]}{m-\zeta} .
$$

Denote $\mathbf{T}_{y}:=\inf \left\{t: X_{t} \geq m\right\}$. Then, since $X$ is a submartingale,

$$
\mathbb{E}\left[\left(m-X_{T}\right) \mathbf{1}_{\left\{X_{T}^{*} \geq m\right\}}\right]=\mathbb{E}\left[\mathbb{E}\left\{m-X_{T} \mid \mathcal{F}_{\mathbf{T}_{m}}\right\} \mathbf{1}_{\left\{\mathbf{T}_{m} \geq T\right\}}\right] \leq \mathbb{E}\left[\left(m-X_{\mathbf{T}_{m}}\right) \mathbf{1}_{\left\{\mathbf{T}_{m} \geq T\right\}}\right] \leq 0 .
$$

Inspecting the last proof, we see that a necessary condition in order to restore equality is that $\mathbb{E}[(m-$ $\left.\left.X_{T}\right) \mathbf{1}_{\left\{X_{T}^{*} \geq m\right\}}\right]=0$, which requires $X$ to be a continuous martingale. Next, to obtain the best possible inequality, we further minimize over $\zeta<m$. Denoting by $\mu$ the marginal distribution of $X_{T}$, we are then reduced to the minimization problem:

$$
\inf _{\zeta<m} f(\zeta) \text { where } f(\zeta):=\frac{\int(x-\zeta)^{+} \mu(d x)}{m-\zeta}
$$

We directly compute that

$$
f^{\prime}(\zeta)=\frac{\int_{[\zeta, \infty)} x \mu(d x)-m \int_{[\zeta, \infty)} \mu(d x)}{(m-\zeta)^{2}}
$$

so that the first order condition for this minimization problem is:

$$
m=b_{\mu}\left(\xi_{m}\right) \quad \text { where } \quad b_{\mu}(x):=\mathbf{1}_{\{\mu([x, \infty)>0\}} \frac{\int_{[x, \infty)} y \mu(d y)}{\int_{[x, \infty)} \mu(d y)}+x \mathbf{1}_{\{\mu([x, \infty)=0\}}
$$

is the barycenter function of the probability measure $\mu$. We also verify immediately that $d f^{\prime}\left(\xi_{m}\right)=\frac{d \mu\left(\xi_{m}\right)}{m-\xi_{m}} \geq 0$ which shows that $\xi_{m}$ is indeed a minimizer.

The barycenter function $b_{\mu}$ is positive, nondecreasing, and continuous whenever $\mu$ has no atoms. Hence the above minimization problem is solved by

$$
\xi_{m}:=b_{\mu}^{-1}(m) \quad \text { where } \quad b_{\mu}^{-1} \text { is the right-continuous inverse of } b_{\mu} .
$$

A pictorial representation of the barycenter function $b_{\mu}$ was introduced by Hobson [18]. In the simple situation where $\mu$ has no atoms, $b_{\mu}(\zeta)$ is the $x$-coordinate of the point where the tangent at $\zeta$ of the function $c_{\mu}(\zeta):=$ $\int(x-\zeta)^{+} \mu(d x)$ intersects the $x$-axis. If $\mu$ has atoms, then the barycenter function is discontinuous, and the last pictorial representation is adapted suitably to this situation.

The following result shows that the Azéma-Yor solution of the SEP reaches the upper bound of Corollary 3.2 . 
Proposition 3.3. Let $\mu$ be a centered probability measure on $\mathbb{R}$, and $\hat{X}_{t}:=B_{\tau^{\mathrm{AY}} \wedge \frac{t}{T-t}}, t \leq T$, where $B$ is a Brownian motion and $\tau^{\mathrm{AY}}:=\inf \left\{t \geq 0: B_{t} \leq b_{\mu}^{-1}\left(B_{t}^{*}\right)\right\}$. Then, for all càdlàg submartingale $X$ with $X_{T} \sim \mu$ :

$$
\mathbb{E}\left[\mathbf{1}_{\left\{\hat{X}_{T}^{*} \geq m\right\}}\right]=\frac{\int\left(x-b_{\mu}^{-1}(m)\right)^{+} \mu(d x)}{m-b_{\mu}^{-1}(m)} \geq \mathbb{E}\left[\mathbf{1}_{\left\{X_{T}^{*} \geq m\right\}}\right] \text { for all } m \geq 0 .
$$

More generally, for all nondecreasing $\phi$, and all càdlàg submartingale $X$ with $X_{T} \sim \mu$ :

$$
\mathbb{E}\left[\phi\left(\hat{X}_{T}^{*}\right)\right]=\phi\left(X_{0}\right)+\int_{X_{0}}^{\infty} d \phi(m) \frac{\int\left(x-b_{\mu}^{-1}(m)\right)^{+} \mu(d x)}{m-b_{\mu}^{-1}(m)} \geq \mathbb{E}\left[\phi\left(X_{T}^{*}\right)\right] .
$$

Proof. (in the case of an atomless $\mu$ ) As in the previous proofs, the second claim follows from the first one due to the non-decrease of $\phi$. We then focus on the first claim.

Given that $b_{\mu}^{-1}$ minimizes the function $f$ in (3.2), the right-hand side inequality is a consequence of Corollary 3.2. So we only need to prove the left-hand side equality.

By the definition of $\hat{X}$, notice that $\hat{X}_{T}^{*} \geq y$ if and only if $X_{T} \geq b_{\mu}^{-1}(y)$. Then

$$
\mathbb{E}\left[\mathbf{1}_{\left\{\hat{X}_{T}^{*} \geq m\right\}}\right]=\mathbb{P}\left[X_{T} \geq b_{\mu}^{-1}(m)\right]=\int_{\left[b_{\mu}^{-1}(m), \infty\right)} \mu(d x)
$$

Moreover, by the definition of $b_{\mu}$, we have $\int_{\left[b_{\mu}^{-1}(m), \infty\right)}(x-m) \mu(d x)=0$. Then:

$$
\mathbb{E}\left[\mathbf{1}_{\left\{\hat{X}_{T}^{*} \geq m\right\}}\right]=\frac{\int_{\left[b_{\mu}^{-1}(m), \infty\right)}\left[\left(m-b_{\mu}^{-1}(m)\right)+(x-m)\right] \mu(d x)}{m-b_{\mu}^{-1}(m)}=\frac{\int\left(x-b_{\mu}^{-1}(m)\right)^{+} \mu(d x)}{m-b_{\mu}^{-1}(m)} .
$$

We conclude this section by providing a financial interpretation of Lemma 3.1:

$$
\mathbf{1}_{\left\{\omega_{T}^{*} \geq m\right\}} \leq \frac{\left(\omega_{T}-\zeta\right)^{+}}{m-\xi}+\frac{m-\omega_{T}}{m-\zeta} \mathbf{1}_{\left\{\omega_{T}^{*} \geq m\right\}} \text { for all } \quad \zeta<m
$$

The left-hand side is the payoff of a Digital option on the running maximum. The right-hand side exhibits a model-free semi-static hedging strategy with the following two components:

- Static hedge: the first term on the right-hand side corresponds to the outcome of $\frac{1}{m-\zeta}$ units of $T$-maturity European calls with strike $\zeta$.

- Dynamic hedge: the second term on the right-hand side corresponds to the dynamic hedging strategy $\frac{m-\omega_{T}}{m-\zeta} \mathbf{1}_{\left\{\omega_{T}^{*} \geq m\right\}}=\int_{0}^{T} H_{t} d \omega_{t}$ with a simple $H$, i.e. piecewise constant. Consequently, the stochastic integral is well-defined in the obvious sense. This dynamic hedging strategy consists in selling $\frac{1}{m-\zeta}$ shares of underlying at time $\mathbf{T}_{m}$ whenever $\mathbf{T}_{m} \leq T$.

A similar financial interpretation holds for a Lookback option with payoff $\phi\left(\omega_{T}^{*}\right)$ for some non-decreasing function $\phi$.

\section{Multi-marginals Extension}

In this section, we present the extension of the results of the previous section to the context of $n$-marginals constraints. The main question is to find the corresponding pathwise inequality, as in Lemma 3.1, so as equality holds for some convenient choice of a martingale. 


\subsection{The two-marginals constraint case}

The following inequality was introduced by Brown, Hobson \& Rogers [7].

Lemma 4.1. Let $\omega: \mathbb{R}_{+} \longrightarrow \mathbb{R}$ be an arbitrary càdlàg function, and denote $\omega_{t}^{*}:=\sup _{s \leq t} \omega_{s}$. Then, for all $\zeta_{1}<\zeta_{2}<m$ :

$$
\begin{aligned}
\mathbf{1}_{\left\{\omega_{t_{2}}^{*} \geq m\right\}} \leq & \frac{\left(\omega_{t_{2}}-\zeta_{2}\right)^{+}}{m-\zeta_{2}}+\frac{\left(\omega_{t_{1}}-\zeta_{1}\right)^{+}}{m-\zeta_{1}}-\frac{\left(\omega_{t_{1}}-\zeta_{2}\right)^{+}}{m-\zeta_{2}} \\
& +\mathbf{1}_{\left\{m \leq \omega_{t_{1}}^{*}\right\}} \frac{m-\omega_{t_{1}}}{m-\zeta_{1}}+\mathbf{1}_{\left\{\omega_{t_{1}}^{*} \geq m, \omega_{t_{1}} \geq \zeta_{2}\right\}} \frac{\omega_{t_{1}}-\omega_{t_{2}}}{m-\zeta_{2}}+\mathbf{1}_{\left\{\omega_{t_{1}}^{*}<m \leq \omega_{t_{2}}^{*}\right\}} \frac{m-\omega_{t_{2}}}{m-\zeta_{2}}
\end{aligned}
$$

Proof. The inequality is easily verified by considering separately each case. We shall prove a more general result in Lemma 4.3 below.

Similar to the previous section, we may deduce from Lemma 4.1 a pathwise inequality for a payoff of the form $\phi\left(\omega_{T}^{*}\right)$ for any non-decreasing $\phi$. We leave the details for the reader. The next result shows the optimality of the previous pathwise inequality.

Proposition 4.2. For all right-continuous martingale $M$ :

$$
\mathbb{P}\left[M_{t_{2}}^{*} \geq y\right] \leq \inf _{\zeta_{1}<\zeta_{2}<m}\left\{\frac{\mathbb{E}\left[\left(M_{t_{2}}-\zeta_{2}\right)^{+}\right]}{m-\zeta_{2}}+\frac{\mathbb{E}\left[\left(M_{t_{1}}-\zeta_{1}\right)^{+}\right]}{m-\zeta_{1}}-\frac{\mathbb{E}\left[\left(M_{t_{1}}-\xi_{2}\right)^{+}\right]}{m-\zeta_{2}}\right\}
$$

Moreover, for all probability measures $\mu_{1} \preceq \mu_{2}$ in the convex order, the exists a martingale $\hat{M}$ such that $\hat{M}_{t_{1}} \sim \mu_{1}, \hat{M}_{t_{2}} \sim \mu_{2}$, and equality holds in the previous inequality

The first part of the proposition follows by taking expectations in the pathwise inequality of Lemma 4.1. The second part is proved by extending the Azéma-Yor solution of the Skorohod embedding problem. we refer to Brown, Hobson and Rogers [7] for this construction.

We finally provide a financial interpretation of the pathwise inequality of Lemma 4.1 in terms of model-free superhedging. Firs, the left-hand side is the payoff of a Digital option on the running maximum. As for the right-hand side, we have the following interpretation of each term:

- The first line exhibits a linear combination of European options with maturities $t_{1}, t_{2}$, and strikes $\zeta_{1}, \zeta_{2}$.

- The term $\mathbf{1}_{\left\{m \leq \omega_{t_{1}}^{*}\right\}} \frac{m-\omega_{t_{1}}}{m-\zeta_{1}}$ corresponds to a simple trading strategy to be implemented at time $\mathbf{T}_{m}$, whenever $\mathbf{T}_{m} \leq t_{1}$.

- The term $\mathbf{1}_{\left\{\omega_{t_{1}}^{*} \geq m, \omega_{t_{1}} \geq \zeta_{2}\right\}} \frac{\omega_{t_{1}}-\omega_{t_{2}}}{m-\zeta_{2}}$ corresponds to a simple trading strategy at time $t_{1}$.

- The term $\mathbf{1}_{\left\{\omega_{t_{1}}^{*}<m \leq \omega_{t_{2}}^{*}\right\}} \frac{m-\omega_{t_{2}}}{m-\zeta_{2}}$ corresponds to a simple trading strategy to be implemented at time $\mathbf{T}_{m}$, whenever $\mathbf{T}_{m} \in\left(t_{1}, t_{2}\right]$.

\subsection{The $n$-marginals constraints case}

An intriguing question is how to guess a pathwise inequality as in Lemmas 3.1 and 4.1. Indeed, despite the obvious verification of these inequalities, one has to come up with an interesting inequality which is sharp in the sense that it is attained by some choice of a martingale. The answer to this question will be provided in Section 5 where an optimal transport approach will be introduced. The efficiency of the optimal transport approach is outlined by the following extension of the pathwise inequality. 
Lemma 4.3. Let $\omega$ be a càdlàg path and denote $\omega_{t}^{*}:=\sup _{0 \leq s \leq t} \omega_{s}$. Then, for $m>\omega_{0}$ and $\zeta_{1} \leq \cdots \leq \zeta_{n}<m$ :

$$
\begin{aligned}
\mathbf{1}_{\omega_{t_{n}}^{*} \geq m} \leq & \sum_{i=1}^{n}\left(\frac{\left(\omega_{t_{i}}-\zeta_{i}\right)^{+}}{m-\zeta_{i}}+\mathbf{1}_{\omega_{t_{i-1}}^{*}<m \leq \omega_{t_{i}}^{*}} \frac{m-\omega_{t_{i}}}{m-\zeta_{i}}\right) \\
& -\sum_{i=1}^{n-1}\left(\frac{\left(\omega_{t_{i}}-\zeta_{i+1}\right)^{+}}{m-\zeta_{i+1}}+\mathbf{1}_{\left\{m \leq \omega_{t_{i}}^{*}, \zeta_{i+1} \leq \omega_{t_{i}}\right\}} \frac{\omega_{t_{i+1}}-\omega_{t_{i}}}{m-\zeta_{i+1}}\right) .
\end{aligned}
$$

Proof. Denote by $A_{n}$ the right-hand side of (4.1), and let us prove the claim by induction. First, in the case $n=1$, the required inequality is the same as that of Lemma 2.1 in [7]:

$$
A_{1}=\frac{\left(\omega_{t_{1}}-\zeta_{1}\right)^{+}+\mathbf{1}_{\left\{\omega_{t_{0}}^{*}<m \leq \omega_{t_{1}}^{*}\right\}}\left(m-\omega_{t_{1}}\right)}{m-\zeta_{1}} \geq \frac{\omega_{t_{1}}-\zeta_{1}+m-\omega_{t_{1}}}{m-\zeta_{1}} \mathbf{1}_{\left\{m \leq \omega_{t_{1}}^{*}\right\}} \geq \mathbf{1}_{\left\{m \leq \omega_{t_{1}}^{*}\right\}}
$$

We next assume that $A_{n-1} \geq \mathbf{1}_{\left\{\omega_{t_{n-1}}^{*} \geq m\right\}}$ for some $n \geq 2$, and show that $A_{n} \geq \mathbf{1}_{\left\{\omega_{t_{n}}^{*} \geq m\right\}}$. We consider two cases.

Case 1: $\omega_{t_{n-1}}^{*} \geq m$. Then $\omega_{t_{n}}^{*} \geq m$, and it follows from the induction hypothesis that $1=\mathbf{1}_{\left\{\omega_{t_{n}}^{*} \geq m\right\}}=$ $\mathbf{1}_{\left\{\omega_{\left.t_{n-1}\right\}}^{*} \geq m\right.} \leq A_{n-1}$. In order to see that $A_{n-1} \leq A_{n}$, we compute directly that, in the present case,

$$
A_{n}-A_{n-1}=\frac{\omega_{t_{n}}-\zeta_{n}}{m-\zeta_{n}}\left(\mathbf{1}_{\left\{\omega_{t_{n}} \geq \zeta_{n}\right\}}-\mathbf{1}_{\left\{\omega_{t_{n-1}} \geq \zeta_{n}\right\}}\right) \geq 0
$$

Case 2: $\omega_{t_{n-1}}^{*}<m$. As $\left(\omega_{t}^{*}\right)$ is non-decreasing, it follows that $\omega_{t_{i}}^{*}<m$ for all $i \leq n-1$. With a direct computation we obtain:

$$
A_{n}=A_{n}^{0}+\frac{\left(\omega_{t_{n}}-\zeta_{n}\right)^{+}}{m-\zeta_{n}}+\mathbf{1}_{\left\{m \leq \omega_{t_{n}}^{*}\right\}} \frac{m-\omega_{t_{n}}}{m-\zeta_{n}}, \text { where } A_{n}^{0}:=\sum_{i=1}^{n-1}\left(\frac{\left(\omega_{t_{i}}-\zeta_{i}\right)^{+}}{m-\zeta_{i}}-\frac{\left(\omega_{t_{i}}-\zeta_{i+1}\right)^{+}}{m-\zeta_{i+1}}\right) .
$$

Since $m>\omega_{t_{i}}^{*} \geq \omega_{t_{i}}$ for $i \leq n-1$, the functions $\zeta \longmapsto\left(\omega_{t_{i}}-\zeta\right)^{+} /(m-\zeta)$ are non-increasing. This implies that $A_{n}^{0} \geq 0$ by the fact that $\zeta_{i} \leq \zeta_{i+1}$ for all $i \leq n$. Then:

$$
\begin{aligned}
A_{n} \geq \frac{\left(\omega_{t_{n}}-\zeta_{n}\right)^{+}+\mathbf{1}_{\left\{m \leq \omega_{t_{n}}^{*}\right\}}\left(m-\omega_{t_{n}}\right)}{m-\zeta_{n}} & \geq \frac{\left(\omega_{t_{n}}-\zeta_{n}\right)^{+}+m-\omega_{t_{n}}}{m-\zeta_{n}} \mathbf{1}_{\left\{m \leq \omega_{t_{n}}^{*}\right\}} \\
& \geq \frac{\omega_{t_{n}}-\zeta_{n}+m-\omega_{t_{n}}}{m-\zeta_{n}} \mathbf{1}_{\left\{m \leq \omega_{t_{n}}^{*}\right\}}=\mathbf{1}_{\left\{m \leq \omega_{t_{n}}^{*}\right\}} .
\end{aligned}
$$

Corollary 4.4. Let $X$ be a càdlàg submartingale, and $\phi$ a nondecreasing function on $\mathbb{R}$. Then, for all functions $\zeta_{1}(m) \leq \ldots \leq \zeta_{n}(m) \leq m$ :

$$
\mathbb{E}\left[\phi\left(X_{t_{n}}^{*}\right)\right] \leq \phi\left(X_{0}\right)-\sum_{i=1}^{n} \int_{X_{0}}^{\infty}\left(\frac{\mathbb{E}\left[\left(X_{t_{i}}-\zeta_{i}(m)\right)^{+}\right]}{m-\zeta_{i}(m)}-\frac{\mathbb{E}\left[\left(X_{t_{i}}-\zeta_{i+1}(m)\right)^{+}\right]}{m-\zeta_{i+1}(m)} \mathbf{1}_{\{i<n\}}\right) d \phi(m) .
$$

Proof. Use the pathwise inequality along the paths of $X$, and take expected values yields the required result for $\phi(x):=\mathbf{1}_{x \geq y}$ for all $y \in \mathbb{R}$. The extension to a general non-decreasing function $\phi$ follows from the same argument as in Corollary 3.1. 
The bound derived in Corollary 4.4 is complemented by an optimality result in Brown, Hobson and Rogers [7] in the two-marginals case $n=2$, and by Henry-Labordère, Obloj, Spoida and Touzi [17] in the present context of $n$ marginals.

We conclude this section by the interpretation of the pathwise inequality of Lemma 4.3 in terms of model-free superhedging of the Digital option on the running maximum defined by the payoff $\xi=\mathbf{1}_{\left\{X_{T}^{*} \geq m\right\}}$. The right hand side of (4.1) consists of three elements: a static position in call options, a forward transaction (with the shortest available maturity) when the barrier $m$ is hit and rebalancing thereafter at times $t_{i}$. More precisely:

(i) Static position in calls:

$$
\sum_{i=1}^{n}\left(\frac{\left(X_{t_{i}}-\zeta_{i}\right)^{+}}{m-\zeta_{i}}-\mathbf{1}_{\{i<n\}} \frac{\left(X_{t_{i}}-\zeta_{i+1}\right)^{+}}{m-\zeta_{i+1}}\right)
$$

For $1 \leq i<n$, we hold a portfolio long and short calls with maturity $t_{i}$ and strikes $\zeta_{i}$ and $\zeta_{i+1}$ respectively. This yields a "tent like" payoff which becomes negative only if the underlying exceeds level $m$. Note that by setting $\zeta_{i}=\zeta_{i+1}$ we may avoid trading the $t_{i}-$ maturity calls. For maturity $t_{n}$ we are only long in a call with strike $\zeta_{n}$.

(ii) Forward transaction if the barrier $m$ is hit: $\mathbf{1}_{\left\{X_{t_{i-1}}^{*}<m \leq X_{t_{i}}^{*}\right\}} \frac{m-X_{t_{i}}}{m-\zeta_{i}}$

At the moment when the barrier $m$ is hit, say between maturities $t_{i^{*}-1}$ and $t_{i^{*}}$, we enter into forward contracts with maturity $t_{i^{*}}$.

Note that the long call position with maturity $t_{i *}$ together with the forward then superhedge the knock-in digital barrier option, cf. (4.3). This resembles the robust semi-static hedge in the one-marginal case, cf. [7, Lemma 2.4]. All the "tent like" payoffs up to maturity $t_{i^{*}-1}$ are non-negative.

(iii) Rebalancing of portfolio to hedge calendar spreads: $-\sum_{i=1}^{n-1} \mathbf{1}_{\left\{m \leq X_{t_{i}}^{*}, \zeta_{i+1} \leq X_{t_{i}}\right\}} \frac{X_{t_{i+1}}-X_{t_{i}}}{m-\zeta_{i+1}}$

After the barrier $m$ was hit, we start trading at times $t_{i}$ in such a way that a potential negative payoff of the calendar spreads $\frac{\left(X_{t_{i+1}}-\zeta_{i+1}\right)^{+}}{m-\zeta_{i+1}}-\frac{\left(X_{t_{i}}-\zeta_{i+1}\right)^{+}}{m-\zeta_{i+1}}, i^{*} \leq i \leq n$, is offset, cf. (4.2).

\section{Optimal Transport Approach to Robust Hedging}

Let $\xi$ be an $\mathcal{F}_{1}$-measurable random variable. Our objective is to formulate the problem of robust superhedging of $\xi$ by means of dynamic trading strategies, in the non risky and the risky assets, and static strategies in $T$-maturity European call and put options.

\subsection{Stochastic integration under a non-dominated family of probability measures}

A dynamic strategy is defined as a predictable process $H$, where $H_{t}$ indicates the number of shares of risky assets to be held at time $t$. Then, the value of a self-financing portfolio at each time $t$ is given by the stochastic integral

$$
Y_{t}^{H}:=Y_{0}+\int_{0}^{t} H_{s} d X_{s}
$$

At this point, we have to discuss a major difficulty raised by the well-posedness of the last stochastic integral. Indeed, on one hand, such a stochastic integral is defined almost surely under some specific measure under which the canonical process $X$ is a semimartingale. On the other hand, the robust feature of superhedging problem imposes that there is no fixed probability measure which determines the distribution of $X$.

To solve this well-posedness problem, we introduce a set of martingale probability measures. Let $\mathbb{P}_{0}$ be the Wiener measure on $\Omega$. For all process $\sigma$ with $\int_{0}^{T} \sigma_{t}^{2} d t<\infty, \mathbb{P}_{0}-$ a.s., we introduce the measure

$$
\mathbb{P}^{\sigma}:=\mathbb{P}_{0} \circ\left(X^{\sigma}\right)^{-1} \quad \text { where } \quad X_{t}^{\sigma}:=X_{0}+\int_{0}^{t} \sigma_{s} d X_{s}, \mathbb{P}_{0}-\text { a.s. }
$$


i.e. $\mathbb{P}^{\sigma}$ is the distribution of the process $X^{\sigma}$ under $\mathbb{P}_{0}$. We further introduce the set

$$
\mathcal{M}^{S}:=\left\{\mathbb{P}^{\sigma}: \int_{0}^{T} \sigma_{t}^{2} d t<\infty, \mathbb{P}_{0}-\text { a.s. and } X^{\sigma} \text { is } \mathbb{P}_{0} \text {-martingale }\right\} .
$$

For all $\mathbb{P} \in \mathcal{M}^{S}$, we denote by $\mathbb{H}_{\text {loc }}^{2}(\mathbb{P})$ the collection of all predictable processes $H$ with $\int_{0}^{T} H_{t}^{2} d t<\infty, \mathbb{P}$-a.s. Clearly the stochastic integral $Y^{H}$ in (5.1) is well-defined for all $H \in \cap_{\mathbb{P} \in \mathcal{M}_{S}} \mathbb{H}_{\text {loc }}^{2}(\mathbb{P})$. In order to avoid dubbling strategies, we now define the set of dynamic hedging strategies as:

$$
\mathcal{H}:=\left\{H \in \cap_{\mathbb{P} \in \mathcal{M}_{S}} \mathbb{H}_{\text {loc }}^{2}(\mathbb{P}): H \text { is } \mathbb{P} \text {-supermartingale, for all } \mathbb{P} \in \mathcal{M}^{S}\right\} \text {. }
$$

\subsection{Robust superhedging and martingale optimal transport}

The set of admissible static positions is:

$$
\Lambda^{\mu}:=\left\{\lambda \in \mathbb{L}^{1}(\mu): \sup _{\mathbb{P} \in \mathcal{M}^{S}} \mathbb{E}^{\mathbb{P}}\left[\lambda\left(X_{T}\right)^{-}\right]<\infty\right\} .
$$

Then, the robust super hedging problem is defined by For all $\lambda \in \Lambda^{\mu}$, we denote $\xi^{\lambda}:=\xi-\lambda\left(X_{1}\right)$. For technical resons, w need to restrict the set $\mathcal{M}^{S}$ (and enlarge the set $\mathcal{H}$ ) as follows:

$$
\begin{aligned}
\mathcal{M}^{S}\left(\xi^{\lambda}\right) & :=\left\{\mathbb{P} \in \mathcal{M}^{S}: \mathbb{E}^{\mathbb{P}}\left[\left(\xi^{\lambda}\right)^{-}\right]<\infty\right\}, \\
\mathcal{H}\left(\xi^{\lambda}\right) & :=\left\{H \in \cap_{\mathbb{P} \in \mathcal{M}^{S}\left(\xi^{\lambda}\right)} \mathbb{H}_{\text {loc }}^{2}(\mathbb{P}): H \text { is } \mathbb{P} \text {-supermartingale, for all } \mathbb{P} \in \mathcal{M}^{S}\left(\xi^{\lambda}\right)\right\} .
\end{aligned}
$$

We are now ready for the definition of the robust super hedging problem:

$$
U^{\mu}(\xi):=\inf \left\{Y_{0}: \exists \Lambda^{\mu} \text { and } H \in \mathcal{H}\left(\xi^{\lambda}\right), Y_{1}^{H} \geq \xi^{\lambda}, \mathcal{M}^{S}\left(\xi^{\lambda}\right)-\text { q.s. }\right\} .
$$

where $\mathcal{M}^{S}\left(\xi^{\lambda}\right)$ - q.s. (quasi-surely) means $\mathbb{P}$-a.s. for all $\mathbb{P} \in \mathcal{M}^{S}\left(\xi^{\lambda}\right)$.

The following result, proved in Possamaï, Royer and Touzi [28], relates the robust super hedging problem to the martingale transport problem.

Theorem 5.1. Let $\xi$ be an $\mathcal{F}_{T}$-measurable r.v. with $\sup _{\mathbb{P} \in \mathcal{M}^{S}} \mathbb{E}^{\mathbb{P}}\left[\xi^{+}\right]<\infty$. Then:

$$
U^{\mu}(\xi)=\inf _{\lambda \in \Lambda^{\mu}} \sup _{\mathbb{P} \in \mathcal{M}^{S}}\left\{\mu(\lambda)+\mathbb{E}^{\mathbb{P}}\left[\xi-\lambda\left(X_{T}\right)\right]\right\} .
$$

Under some additional regularity conditions on the random variable $\xi$, Dolinsky and Soner [15] prove in addition that

$$
U^{\mu}(\xi)=\inf _{\lambda \in \Lambda^{\mu}} \sup _{\mathbb{P} \in \mathcal{M}^{S}}\left\{\mu(\lambda)+\mathbb{E}^{\mathbb{P}}\left[\xi-\lambda\left(X_{T}\right)\right]\right\}=\sup _{\mathbb{P} \in \mathcal{M}^{S}(\mu)} \mathbb{E}^{\mathbb{P}}[\xi]
$$

where $\mathcal{M}^{S}(\mu)$ is the collection of all measures $\mathbb{P} \in \mathcal{M}^{S}$ such that $X_{T} \sim_{\mathbb{P}} \mu$. Under this form, we now see clearly that the robust superhedging reduces to an optimal transport problem on the canonical space of paths with coupling function defined by the payoff of the derivative security, and under a martingale constraint on the coupling measures. The last constraint, which is not present in standard optimal transport, is the main novelty introduced by the robust super hedging problem.

Remark 5.2. The dual formulation of Proposition 5.1 gives access to the optimal hedging strategy and the worst case model. This requires to prove an additional existence result of a solution to the inf-sup problem $\left(\lambda^{*}, \mathbb{P}^{*}\right)$. Then $\lambda^{*}$ is the optimal $T$-maturity Vanilla profile, and $\mathbb{P}^{*}$ is the worst case model corresponding to the upper bound. The optimal dynamic hedging strategy in the underlying asset is as usual obtained by representation of the residual security $\xi-\lambda^{*}\left(X_{T}\right)$. 
Remark 5.3. The dual formulation of Proposition 5.1 is suitable for numerical approximation. Indeed, for each fixed multiplier $\lambda$, the maximization problem is a (singular) stochastic control problem which may be approximated by finite differences or Monte Carlo methods. The optimization stage with respect to $\lambda$ requires an additional iteration. This issue is addressed in Bonnans and Tan [5] and Tan and Touzi [29].

\section{Application to LOOKBACK Options}

Throughout this section, we consider the Lookback option example $\xi=\phi\left(X_{T}^{*}\right)$, as in (3.1), in the one dimensional case $d=1$. We recall that the distribution of $X_{T}$ is the fixed probability measure $\mu$, and we assume in addition that $\phi$ is $C^{1}$ and nondecreasing.

\subsection{The one-marginal case}

Our main interest is to show that the optimal upper bound given by Proposition 5.1:

$$
U^{\mu}(\xi)=\inf _{\lambda \in \Lambda^{\mu}}\left\{\mu(\lambda)+u^{\lambda}\left(0, X_{0}, X_{0}\right)\right\}
$$

reproduces the bound corresponding to the Azema-Yor solution to the Skorohod embedding problem, as shown by Proposition 3.3. Here, $u^{\lambda}$ is the value function of the dynamic version of the stochastic control problem

$$
u^{\lambda}(t, x, m):=\sup _{\mathbb{P} \in \mathcal{P}_{\infty}} \mathbb{E}^{\mathbb{P}}\left[\phi\left(M_{T}^{t, x, m}\right)-\lambda\left(X_{T}^{t, x}\right)\right], \quad t \leq T, \quad(x, m) \in \boldsymbol{\Delta},
$$

where $\left.\boldsymbol{\Delta}:=\left\{(x, m) \in \mathbb{R}^{2}: x \leq m\right\}, X_{u}^{t, x}, u \in[t, T]\right\}$ is the canonical process on the shifted canonical space started from $(t, x)$, and

$$
M_{u}^{t, x, m}:=m \vee \max _{t \leq r \leq u} X_{r}^{t, x}, \quad 0 \leq t \leq u \leq T .
$$

When the time origin is zero, we shall simply write $X_{u}^{x}:=X_{u}^{0, x}$ and $M_{u}^{x, m}:=M_{u}^{0, x, m}$.

By standard time change techniques, we may convert the optimization problem $u^{\lambda}$ into the infinite horizon optimal stopping problem:

$$
u^{\lambda}(x, m)=\sup _{\tau \in \mathcal{T}_{\infty}} \mathbb{E}^{\mathbb{P}_{0}}\left[\phi\left(M_{\tau}^{x, m}\right)-\lambda\left(X_{\tau}^{x}\right)\right] \quad \text { for all } \quad(x, m) \in \boldsymbol{\Delta},
$$

where $\mathcal{T}_{\infty}$ is the collection of all stopping times $\tau$ such that the stopped process $\left\{X_{t \wedge \tau}, t \geq 0\right\}$ is a $\mathbb{P}_{0}-$ uniformly integrable martingale. Then, the set $\Lambda^{\mu}$ of (5.2) translates to:

$$
\Lambda_{0}^{\mu}=\left\{\lambda \in \mathbb{L}^{1}(\mu): \sup _{\tau \in \mathcal{T}_{\infty}} \mathbb{E}\left[\lambda\left(X_{\tau}\right)^{-}\right]<\infty\right\}
$$

By the definition of the dual problem $U^{\mu}$ in $(6.1)$, notice that:

$$
U^{\mu}(\xi) \geq \mathbb{E}^{\mathbb{P}_{0}}\left[\phi\left(M_{\tau}^{x, m}\right)\right] \quad \text { for all solution } \tau \text { of } \operatorname{SEP}(\mu) .
$$

Our objective is to prove that equality is achieved with the Azéma-Yor solution of the SEP.

1. We start from a guess of the value function $u^{\lambda}$ for functions $\lambda$ in the subset:

$$
\hat{\Lambda}_{0}^{\mu}:=\left\{\lambda \in \Lambda_{0}^{\mu}: \lambda \text { is convex }\right\}
$$

By classical tools from stochastic control theory, the value function $u^{\lambda}(x, m)$ is expected to solve the dynamic programming equation:

$$
\min \left\{u^{\lambda}-\phi+\lambda,-u_{x x}^{\lambda}\right\}=0 \text { on } \boldsymbol{\Delta} \text { and } u_{m}^{\lambda}(m, m)=0 \text { for } m \in \mathbb{R} .
$$


The first part of the above DPE is an ODE for which $m$ appears only as a parameter involved in the domain on which the ODE must hold. Since we are restricting to convex $\lambda$, one can guess a solution of the form:

$$
v^{\psi}(x, m):=\phi(m)-\lambda(x \wedge \psi(m))-\lambda^{\prime}(\psi(m))(x-x \wedge \psi(m))
$$

i.e. $v^{\psi}(x, m)=\phi(m)-\lambda(x)$ for $x \leq \psi(m)$ and is given by the tangent at the point $\psi(m)$ for $x \in[\psi(m), m]$. For later use, we observe that for $x \in[\psi(m), m]$ :

$$
\begin{aligned}
v^{\psi}(x, m) & =\phi(m)-\lambda(\psi(m))+\int_{\psi(m)}^{x} \frac{\partial}{\partial y}\left\{\lambda^{\prime}(y)(x-y)\right\} d y \\
& =\phi(m)-\lambda(x)+\int_{\psi(m)}^{x}(x-y) \lambda^{\prime \prime}(d y) \text { for } x \in[\psi(m), m]
\end{aligned}
$$

where $\lambda^{\prime \prime}$ is the second derivative measure of the convex function $\lambda$.

We next choose the function $\psi$ in order to satisfy the Neumann condition in (6.6). Assuming that $\lambda$ is smooth, we obtain by direct calculation that the free boundary $\psi$ must verify the ordinary differential equation $(\mathrm{ODE})$ :

$$
\lambda^{\prime \prime}(\psi(m)) \psi^{\prime}(m)=\frac{\phi^{\prime}(m)}{m-\psi(m)} \quad \text { for all } \quad m \in \mathbb{R}
$$

For technical reasons, we need to consider this ODE in the relaxed sense. Since $\lambda$ is convex, its second derivative $\lambda^{\prime \prime}$ is well-defined as measure on $\mathbb{R}_{+}$. We then introduce the weak formulation of the ODE (6.9):

$$
\int_{\psi^{-1}(B)} \lambda^{\prime \prime}(d y)=\int_{B} \frac{\phi^{\prime}(m)}{m-\psi(m)} d m \text { for all } \quad B \in \mathcal{B}(\mathbb{R})
$$

and we introduce the collection of all relaxed solutions of (6.9):

$$
\Psi^{\lambda}:=\{\psi \text { right-continuous : (6.10) holds and } \psi(m)<m \text { for all } m \in \mathbb{R}\}
$$

Then, by a standard verification argument based on Itô's formula, we see that

$$
u^{\lambda} \leq v^{\psi}
$$

Remark 6.1 (Pathwise inequality). The last verification argument is based on Itô's formula which provides:

$$
\phi\left(X_{T}^{*}\right)-\lambda\left(X_{T}\right)=v^{\psi}\left(X_{0}, X_{0}\right)+\int_{0}^{T} v_{x}^{\psi}\left(X_{t}, X_{t}^{*}\right) d X_{t}+\frac{1}{2} \int_{0}^{T} v_{x x}^{\psi}\left(X_{t}, X_{t}^{*}\right) d t+\int_{0}^{T} v_{m}^{\psi}\left(X_{t}^{*}, X_{t}^{*}\right) d X_{t}^{*}
$$

Since $v_{x x}^{\psi} \leq 0$ and $v_{m}^{\psi}(m, m)=0$, this provides the pathwise inequality:

$$
v^{\psi}\left(X_{0}, X_{0}\right)+\int_{0}^{T} v_{x}^{\psi}\left(X_{t}, X_{t}^{*}\right) d X_{t}+\lambda\left(X_{T}\right) \geq \phi\left(X_{T}^{*}\right)
$$

which holds for any $\lambda$ and any solution $\psi$ of the ODE (6.9). The best pathwise inequality is the one induced by the optimal choice of $\psi$, which completely determines the corresponding optimal $\lambda$ by the ODE (6.9). Consequently, the present approach is a constructive method for the pathwise inequality of Lemma 3.1. 
2. Given the expression of $v^{\psi}$ in (6.7), the last inequality implies that

$$
\mu(\lambda)+u^{\lambda}\left(X_{0}, X_{0}\right) \leq \phi\left(X_{0}\right)+\mu(\lambda)-\lambda\left(X_{0}\right)+\int\left(X_{0}-y\right)^{+} \mathbf{1}_{\left\{y \geq \psi\left(X_{0}\right)\right\}} \lambda^{\prime \prime}(d y) .
$$

Denoting $c_{0}(y):=\left(X_{0}-y\right)^{+}$and $c(y):=\int(x-y)^{+} \mu(d x)$, and applying two integrations by parts to the difference $\mu(\lambda)-\lambda\left(X_{0}\right)$, we get:

$$
\begin{aligned}
\mu(\lambda)+u^{\lambda}\left(X_{0}, X_{0}\right) & \leq \phi\left(X_{0}\right)+\int\left(c(y)-c_{0}(y)\left(1_{\left\{y<X_{0}\right\}}-1_{\left\{\psi\left(X_{0}\right)<y<X_{0}\right\}}\right)\right) \lambda^{\prime \prime}(d y) \\
& =\phi\left(X_{0}\right)+\int\left(c(y)-c_{0}(y) 1_{\left\{y<\psi\left(X_{0}\right)\right\}}\right) \lambda^{\prime \prime}(d y) \\
& =\phi\left(X_{0}\right)+\int \frac{c(\psi(m))-c_{0}(\psi(m)) \mathbf{1}_{\left\{m<X_{0}\right\}}}{m-\psi(m)} \phi^{\prime}(m) d m,
\end{aligned}
$$

by the ODE (6.9) satisfied by $\psi$ in the distribution sense. This calculation provides the following bound for $U^{\mu}(\xi)$ :

$$
U^{\mu}(\xi) \leq \phi\left(X_{0}\right)+\inf _{\psi(.)} \int \frac{c(\psi(m))-c_{0}(\psi(m)) \mathbf{1}_{\left\{m<X_{0}\right\}}}{m-\psi(m)} \phi^{\prime}(m) d m
$$

3. Since $g$ is non-decreasing, the right-hand side is easily shown to reduce to the pointwise minimization of the function inside the integral. Recall that $\int x \mu(d x)=X_{0}$. Then, the last minimization provides:

$$
\inf _{\xi<m} \frac{c(\xi)-c_{0}(\xi) \mathbf{1}_{\left\{m<X_{0}\right\}}}{m-\xi}=\frac{c(0)-c_{0}(0) \mathbf{1}_{\left\{m<X_{0}\right\}}}{m}=0 \quad \text { for } \quad m<X_{0},
$$

and, for $m \geq X_{0}$, we are reduced to the same problem as in (3.2), namely:

$$
\inf _{\xi<m} \frac{c(\xi)}{m-\xi}=\frac{c\left(b_{\mu}^{-1}(m)\right)}{m-b_{\mu}^{-1}(m)} \quad \text { for } \quad m \geq X_{0}
$$

where $b_{\mu}^{-1}$ is the right-continuous inverse of the barycenter function $b_{\mu}$. This bound is in fact the so-called Hardy-Littlewood transform of the measure $\mu$ :

$$
\mu^{\mathrm{HL}}\left(([m, \infty)):=\frac{c\left(b_{\mu}^{-1}(m)\right)}{m-b_{\mu}^{-1}(m)}\right.
$$

which is known from to correspond to the distribution of the running maximum of the Brownian motion at the Azéma-Yor stopping, i.e. $B_{\tau_{\mathrm{AY}}} \sim \mu^{\mathrm{HL}}$. Hence:

$$
U^{\mu}(\xi) \leq \phi\left(X_{0}\right)+\int \mu^{\mathrm{HL}}\left(([m, \infty)) \phi^{\prime}(m) d m=\mu^{\mathrm{HL}}(\phi)\right.
$$

\subsection{The case of finitely-many intermediate marginals}

We now adapt our general methodology to the multiple-marginal context. Our objective is to solve the robust superhedging problem for a Lookback derivative security

$$
\phi\left(X_{T}^{*}\right) \text { given the marginals } X_{t_{i}} \sim \mu_{i} \text { for all } i=1, \ldots, n \text {. }
$$


The probability measures $\mu_{i}$ are defined from market prices which do not admit arbitrage, which by classical results imposes the condition:

$$
\left(\mu_{i}\right)_{1 \leq i \leq n} \text { non-decreasing in the convex order. }
$$

Similar to the one-marginal case, the robust superhedging bound can be expressed in the dual formulation of Proposition 5.1 as:

$$
U_{n}^{\mu}(\xi):=\inf _{\lambda \in \Lambda_{n}^{\mu}(\xi)}\left\{\mu(\lambda)+u^{\lambda}\left(X_{0}, X_{0}\right)\right\}
$$

where

$$
u^{\lambda}(x, m):=\sup _{\mathbb{P} \in \mathcal{P}^{*}} \mathbb{E}_{x, m}^{\mathbb{P}}\left[\phi^{\lambda}\left(X_{t_{1}}, \ldots, X_{t_{n}}, M_{t_{n}}\right)\right], \quad \phi^{\lambda}\left(x_{1}, \ldots, x_{n}, m\right):=\phi(m)-\sum_{i=1}^{n} \lambda_{i}\left(x_{i}\right),
$$

and the set of Lagrange multipliers is:

$$
\Lambda_{n}^{\mu}(\xi)=\left\{\lambda=\left(\lambda_{1}, \ldots, \lambda_{n}\right): \lambda_{i} \in \mathbb{L}^{1}\left(\mu_{i}\right) \text { and } \sup _{\mathbb{P} \in \mathcal{P}^{*}} \mathbb{E}^{\mathbb{P}}\left[\phi^{\lambda}\left(X_{t_{1}}, \ldots, X_{t_{n}}, X_{t_{n}}^{*}\right)^{+}\right]<\infty\right\}
$$

Our approach to solve the present $n$-marginals Skorokhod embedding problem is to introduce the sequence of intermediate optimization problems:

$$
u_{n}(x, m)=\phi(m) \quad \text { and } \quad u_{k-1}(x, m)=\sup _{\mathbb{P} \in \mathcal{P}^{*}} \mathbb{E}_{t_{k-1}, x, m}^{\mathbb{P}}\left[u_{k}^{\lambda}\left(X_{t_{k}}, M_{t_{k}}\right)\right], \quad k \leq n,
$$

where $\mathbb{E}_{t_{k-1}, x, m}^{\mathbb{P}}=\mathbb{E}^{\mathbb{P}}\left[\cdot \mid(X, M)_{t_{k-1}}=(x, m)\right]$, and:

$$
u_{k}^{\lambda}(x, m):=u_{k}(x, m)-\lambda_{k}(x) \text { for }(x, m) \in \Delta .
$$

Given this iterative sequence of value functions, it follows from the dynamic programming principle that our problem of interest is given by:

$$
u^{\lambda}=u_{0}^{\lambda} \quad \text { for all } \quad \lambda \in \Lambda_{n}^{\mu}(\xi)
$$

Similar to the one-marginal case, we may convert the stochastic control problem in (6.15) into a sequence of optimal stopping problems:

$$
u_{k-1}(x, m)=\sup _{\tau \in \mathcal{T}^{*}} \mathbb{E}_{x, m}^{\mathbb{P}_{0}}\left[u_{k}^{\lambda}\left(X_{\tau}, M_{\tau}\right)\right]
$$

Then, denoting by $\mathcal{S}_{n}^{*}:=\left\{\tau=\left(\tau_{1}, \ldots, \tau_{n}\right) \in \mathcal{T}^{*}: \tau_{1} \leq \cdots \leq \tau_{n}\right\}$, we see that

$$
U_{n}^{\mu}(\xi)=\inf _{\lambda \in \Lambda_{n}^{\mu}(\xi)}\left\{\mu(\lambda)+u_{0}^{\lambda}\left(X_{0}, X_{0}\right)\right\} \quad \text { where } \quad u_{0}^{\lambda}(x, m):=\sup _{\tau \in \mathcal{S}_{n}^{*}} \mathbb{E}_{x, m}^{\mathbb{P}_{0}}\left[\phi^{\lambda}\left(X_{\tau}, M_{\tau_{n}}\right)\right]
$$

and the set $\Lambda_{n}^{\mu}(\xi)$ of $(6.14)$ translates in the present context to:

$$
\Lambda_{n}^{\mu}(\xi)=\left\{\lambda=\left(\lambda_{1}, \ldots, \lambda_{n}\right): \lambda_{i} \in \mathbb{L}^{1}(\mu) \text { and } \sup _{\tau \in \mathcal{S}_{n}^{*}} \mathbb{E}^{\mathbb{P}_{0}}\left[\phi^{\lambda}\left(X_{\tau}, X_{\tau_{n}}^{*}\right)^{+}\right]<\infty\right\} .
$$

The function $u_{k-1}$ corresponds to the optimization problem considered in the previous section, with a payoff $\phi(x, m)=u_{k}(x, m)$ depending on the state variable $x$ and the corresponding running maximum $m$. We then need to extend the one-marginal problem to this context. 
To solve the multiple marginals problem, we introduce the candidate value functions:

$$
\begin{aligned}
v_{n}(x, m) & :=\phi(m), \quad v_{k}^{\lambda}(x, m):=v_{k}(x, m)-\lambda_{k}(x), \text { and } \\
v_{k-1}(x, m) & :=v_{k}^{\lambda}\left(x \wedge \psi_{k}(m), m\right)+\left(x-\psi_{k}(m)\right)^{+} \partial_{x} v_{k}^{\lambda}\left(\psi_{k}(m), m\right) \\
& =v_{k}^{\lambda}(x, m)-\int_{\psi_{k}(m)}^{x \vee \psi_{k}(m)}(x-\xi) \partial_{x x} v_{k}^{\lambda}(d \xi, m),
\end{aligned}
$$

where $\psi=\left(\psi_{1}, \ldots, \psi_{n}\right)$ with $\psi_{i}$ an arbitrary solution of the ordinary differential equation

$$
-\psi_{k}^{\prime} \partial_{x x} v_{k}^{\lambda}\left(\psi_{k}, m\right)=\gamma_{k}\left(\psi_{k}, m\right), \quad \text { with } \quad \gamma_{k}(x, m):=(m-x) \partial_{x}\left\{\frac{\partial_{m} v_{k}(x, m)}{m-x}\right\},
$$

which stays strictly below the diagonal. Notice that, in contrast to the one-marginal case, we have dropped here the dependence of $v_{k}$ in $\psi$ by simply denoting $v_{k}:=v_{k}^{\psi}$ and $v_{k}^{\lambda}:=v_{k}^{\psi, \lambda}$.

Similar to the one-marginal case, we introduce the weak formulation of this ODE:

$$
\begin{gathered}
\psi_{k}(m)<m \text { for all } m \geq 0, \text { and } \\
-\int_{\psi(E)} \partial_{x x} v_{k}^{\lambda}\left(., \psi_{k}^{-1}\right)(d \xi)=\int_{E} \gamma_{k}\left(\psi_{k}, .\right)(d m) \text { for all } E \in \mathcal{B}(\mathbb{R}),
\end{gathered}
$$

and we introduce the set

$$
\Psi_{n}^{\lambda}:=\left\{\psi: \mathbb{R} \rightarrow \mathbb{R}^{n} \text { with right-continuous entries } \psi_{k} \text { satisfying }(6.22), k \leq n\right\} .
$$

We also follow the one-marginal case by restricting the minimization in (6.18) to those multipliers $\lambda$ in the set:

$$
\hat{\Lambda}_{n}^{\mu}(\xi):=\left\{\lambda \in \Lambda_{n}^{\mu}(\xi): v_{k-1} \text { concave in } x \text { and } v_{k-1} \geq v_{k}^{\lambda} \text { for all } k \leq n\right\} .
$$

Similar to the one-marginal case, we may prove that

$$
u^{\lambda}\left(X_{0}, X_{0}\right) \leq v_{0}\left(X_{0}, X_{0}\right) \text { for all } \lambda \in \hat{\Lambda}_{n}^{\mu} \text { and } \psi \in \Psi_{n}^{\lambda} .
$$

This inequality is derived by an iterative verification argument based on Itô's formula. In particular, similar to Remark 6.1, we obtain as a by-product of the proof a family of pathwise inequalities parameterized by the choice of the Lagrange multipliers $\lambda_{i}$ and the corresponding free boundaries $\psi_{i}$. The optimal choice of $\psi_{i}$ 's determines the optimal choice of $\lambda_{i}$ 's, by (6.21), and induces the sharpest pathwise inequality of Lemma 4.3.

We have then reproduced Step 1 of Subsection 6 in the present context of finitely-many intermediate laws.

The optimization over the Lagrange multipliers (and in fact the corresponding free boundaries $\psi_{i}$ 's) can now be processed as in Steps 2 and 3 of Subsection 6. The analysis in the present context is more involved. Therefore, we refrain from reproducing it, and we refer the interested reader to [17].

\section{REFERENCES}

[1] Acciaio, B., Beiglböck, F., Penkner, F., Schachermayer, W. and Temme, J. (2012), Trajectorial Interpretation of Doob's martingale inequalities. Online First in Ann. Appl. Prob. (2012).

[2] Azéma, J. and Yor, M. (1979), Une solution simple au problème de Skorokhod, Séminaire de Probabilités XIII, 90-115, LNM 721.

[3] Azéma, J. and Yor, M. (1979), Le problème de Skorokhod: complément à l'exposé précédent, Séminaire de Probabilités XIII, 625-633, LNM 721.

[4] Beiglböck, M., Henry-Labordère, P. and Penkner, F. (2011), Model independent Bounds for Option Prices: A Mass Transport Approach. Finance and Stochastics 17, Issue 3, pp 477-501. 
[5] Bonnans, F. and Tan, X. (2011), A model-free no-arbitrage price bound for variance options, to appear in Applied Mathematics and Optimization.

[6] Breeden, D.T. and Litzenberger, R.H. (1978), Prices of state-contingent claims implicit in option prices, J. Business 51, 621-651.

[7] Brown, H., Hobson, D. and Rogers, L.C.G. (1998), The maximum maximum of a martingale constrained by an intermediate law, Probability Theory and Related Fields 119(4), 558-578.

[8] Brown, H., Hobson, D. and Rogers, L.C.G. (2001), Robust hedging of barrier options, Mathematical Finance 11(3), $285-314$.

[9] Bouchard, B. and Nutz, M. (2013), Arbitrage and duality in nondominated discrete-time models. Preprint.

[10] Cox, A. and Hobson, D. (2005), Local martingales, bubbles and option prices, Finance and Stochastics 9(4), 477-492.

[11] Cox, A. M. G.,Hobson, D. and Obłój, J. (2008), Pathwise inequalities for local time: applications to Skorokhod embeddings and optimal stopping, Ann. Appl. Probab. 18 (5), 1870-1896.

[12] Cox, A. and Obłój, J. (2011), Robust pricing and hedging of double no-touch options, Finance and Stochastics 15(3), 573-605.

[13] Cox, A. and Obłój, J. (2011), Robust pricing and hedging of double touch options, SIAM Journal on Financial Mathematics $2,141-182$.

[14] Cox, A. and Wang, J. (2013), Root's barrier: Construction, optimality and applications to variance options, Annals of Applied Probability 23(3), 859-894.

[15] Dolinsky, J. and Soner, M. (2012), Martingale optimal transport and robust hedging in continuous time. Probability Theorey and Related Fields, to appear. Preprint, arXiv:1208.4922.

[16] Galichon, A., Henry-Labordère, P., Touzi, N. (2011), A stochastic control approach to no-arbitrage bounds given marginals, with an application to Lookback options, to appear in Annals of Applied Probability.

[17] Henry-Labordère, P. Obloj, J. Spoida, P. and Touzi, N. (2013). Maximum Maximum of Martingales given Finitely-Many Intermediate Marginals. Preprint.

[18] Hobson, D. (1998), Robust hedging of the Lookback option, Finance and Stochastics 2, 329-347.

[19] Hobson, D. (1998), The maximum maximum of a martingale, Séminaire de Probabilités XXXII, pp250-263.

[20] Hobson, D. (2010), The Skorokhod Embedding Problem and Model-Independent Bounds for Option Price in Paris-Princeton Lectures on Mathematical Finance, Springer, LNM 2003.

[21] Hobson, D. and Klimmek, M. (2010), Maximising functionals of the joint law of the maximum and terminal value in the Skorokhod embedding problem, arXiv:1012.3909, to appear in Annals of Applied Probability.

[22] Hobson, D. and Neuberger, A. (2012), Robust bounds for forward start options, Mathematical Finance, 22, 31-56.

[23] Madan, D.B, Yor, M. (2002), Making Markov martingales meet marginals: with explicit constructions, Bernoulli 8(4), 509-536.

[24] Nutz, M. (2012). Pathwise Construction of Stochastic Integrals. Electronic Communications in Probability 17(24), 1-7.

[25] Neufeld, A. and Nutz, M. (2012), Superreplication under Volatility Uncertainty for Measurable Claims. Preprint, arXiv:1208.6486.

[26] Obłój, J. (2004), The Skorokhod embedding problem and its offspring, Probab. Surv. 1, 321-390 (electronic).

[27] Obłój, J. and Spoida, P. (2013), An Iterated Azéma-Yor Type Embedding for Finitely Many Marginals. Preprint, arXiv:1304.0368.

[28] Possamaï, D., Royer, G. and Touzi, N. (2013). On the Robust Superhedging of Measurable Claims. Preprint, arXiv:1302.1850.

[29] Tan, X. and Touzi, N. (2011), Dual Formulation of the optimal transportation problem under controlled stochastic dynamics. Annals of Probability, to appear. 